\section{OPEN ACCESS}

Edited by:

P. Bryant Chase,

Florida State University, United States

Reviewed by: Javier Rodriguez-Falces,

Escuela de Negocios de Navarra,

Spain

Ryota Akagi,

Shibaura Institute of Technology,

Japan

${ }^{*}$ Correspondence:

Sebastian Garcia-Vicencio sebastian.garciavicencio@gmail.com

${ }^{\dagger}$ Present address:

Emeric Chalchat

Laboratoire AME2P, Université

Clermont Auvergne,

Clermont-Ferrand, France

¥These authors have contributed equally to this work

Specialty section: This article was submitted to

Striated Muscle Physiology, a section of the journal Frontiers in Physiology

Received: 10 December 2019 Accepted: 19 March 2020

Published: 24 April 2020

Citation:

Chalchat E, Gennisson J-L, Peñailillo L, Oger M, Malgoyre A, Charlot K, Bourrilhon C, Siracusa J and Garcia-Vicencio S (2020) Changes in the Viscoelastic Properties of the Vastus Lateralis Muscle With

Fatigue. Front. Physiol. 11:307. doi: 10.3389/fphys.2020.00307

\title{
Changes in the Viscoelastic Properties of the Vastus Lateralis Muscle With Fatigue
}

\begin{abstract}
Emeric Chalchat ${ }^{1 \dagger}$, Jean-Luc Gennisson², Luis Peñailillo ${ }^{3}, M_{y r i a m ~ O g e r} 4$, Alexandra Malgoyre 1,5, Keyne Charlot ${ }^{1,5}$, Cyprien Bourrilhon ${ }^{1,5}$, Julien Siracusa ${ }^{1,5 \neq}$ and Sebastian Garcia-Vicencio ${ }^{1,5 * \neq}$
\end{abstract}

\begin{abstract}
${ }^{1}$ Unité de Physiologie de l'Exercice et des Activités en Conditions Extrêmes, Département Environnements Opérationnels, Institut de Recherche Biomédicale des Armées, Brétigny-sur-Orge, France, ${ }^{2}$ BIOMAPS, Laboratoire d'Imagerie Biomédicale Multi-Modale, CEA, Université Paris-Saclay, CNRS UMR 9011, INSERM UMR 1281, Orsay, France, ${ }^{3}$ Exercise Science Laboratory, School of Kinesiology, Faculty of Medicine, Finis Terrae University, Santiago, Chile, ${ }^{4}$ Unité Imagerie, Département des Plateformes et Recherche Technologique, Institut de Recherche Biomédicale des Armées, Brétigny-sur-Orge, France, ${ }^{5}$ LBEPS, Univ Evry, IRBA, Université Paris Saclay, Evry, France
\end{abstract}

We investigated the in vivo effects of voluntary fatiguing isometric contractions of the knee extensor muscles on the viscoelastic properties of the vastus lateralis (VL). Twelve young males (29.0 \pm 4.5 years) performed an intermittent voluntary fatigue protocol consisting of 6 sets $\times 10$ repetitions of 5-s voluntary maximal isometric contractions with 5-s passive recovery periods between repetitions. Voluntary and evoked torque were assessed before, immediately after, and 20 min after exercise. The shear modulus $(\mu)$ of the VL muscle was estimated at rest and during a ramped isometric contraction using a conventional elastography technique. An index of active muscle stiffness was then calculated (slope from the relationship between shear modulus and absolute torque). Resting muscle viscosity $(\eta)$ was quantified using a shear-wave spectroscopy sequence to measure the shear-wave dispersion. Voluntary and evoked torque decreased by $\sim 37 \%(P<0.01)$ immediately after exercise. The resting $V L \mu$ was lower at the end of the fatigue protocol $(-57.9 \pm 5.4 \%, P<0.001)$, whereas the resting $V L \eta$ increased $(179.0 \pm 123 \%, P<0.01)$. The active muscle stiffness index also decreased with fatigue $(P<0.05)$. By 20 min post-fatigue, there were no significant differences from the preexercise values for $V L \eta$ and the active muscle stiffness index, contrary to the resting $V L \mu$. We show that the VL $\mu$ is greatly reduced and $\eta$ greatly enhanced by fatigue, reflecting a more compliant and viscous muscle. The quantification of both shear $\mu$ and $\eta$ moduli in vivo may contribute to a better understanding of the mechanical behavior of muscles during fatigue in sports medicine, as well as in clinical situations.

Keywords: stiffness, viscosity, exercise, shear-wave spectroscopy, shear-wave elastography, isometric contractions, muscle compliance

\section{INTRODUCTION}

In vivo characterization of the viscoelastic mechanical properties of skeletal muscle [e.g., stiffness or shear modulus $(\mu)$ and shear viscosity $(\eta)$ ] has long been a great interest (Gennisson et al., 2010; Lacourpaille et al., 2012, 2014; Andonian et al., 2016; Davis et al., 2018; Rasool et al., 2018). Recent evidence suggests that the assessment of subtle modifications of muscle stiffness may be 
helpful in identifying the early development of muscle fatigue (Andonian et al., 2016; Siracusa et al., 2019), damage (Lacourpaille et al., 2014), or disease (Rasool et al., 2018). We recently demonstrated (Siracusa et al., 2019) that the resting vastus lateralis $\mu(\mathrm{VL} \mu)$, assessed by shear-wave elastography (SWE), was reduced $(-34.7 \pm 6.7 \%)$ after a series of voluntary isometric contractions of the knee extensor (KE) muscles. Moreover, such reduced muscle stiffness was accompanied by a decrease in the voluntary torque and modification of the mechanical properties of a single twitch (e.g., reduced peak torque, longer electromechanical delay and contraction time, and reduced rate of force development) of the KE after exercise. These results suggest that measured modifications in resting muscle stiffness can be used to complement traditional neuromuscular measurements to estimate changes in the mechanical properties of skeletal muscle with fatigue. Aside from such studies under resting conditions, the study of active muscle stiffness may provide valuable information about patterns of muscle fatigue during exercise. Identifying patterns of muscle fatigue related to changes in muscle stiffness could help diagnose muscle states (e.g., neuromuscular disorders) or provide insights for muscle rehabilitation in athletes. However, conflicting results have been reported. Indeed, decreases in active stiffness with fatigue have been confirmed after dynamic and intermittent submaximal isometric contractions (Vigreux et al., 1980; Morel et al., 2019), but not after sustained isometric protocols (Bouillard et al., 2012a; Akagi et al., 2019). Akagi et al. (2019) showed an increase in resting VL $\mu(21.7 \pm 32.4 \%)$ after a low-intensity prolonged fatiguing task of the KE muscles. Akagi et al. (2017) suggested that resting muscle stiffness could be higher after prolonged fatiguing task due to muscle failure to fully relax and cramps. Moreover, it is not known whether the changes in viscoelastic properties of muscles contribute to restoration of force transmission capacities during the recovery period. The well-known task dependency of fatigue needs to be considered to better understand discrepancies concerning changes in muscle stiffness immediately after fatiguing exercise and during the recovery period.

Studies on muscle viscoelasticity have mainly focused on characterizing elastic behavior, largely neglecting the viscous component. The concept of muscle $\eta$ was first introduced by Hill (1922) and was defined as the resistance (frictional forces or energy dissipative elements) of fluid to flow when it is subjected to shear stress. It has been suggested that the viscous properties of muscle, such as that of the myoplasm, concentrations of inorganic phosphate, and weakly attached cross-bridges, collagen fibrils, titin, and other cytoskeletal proteins (Mauro and Adams, 1961; Moss and Halpern, 1977; Stackhouse et al., 2001), influence the capacity to develop maximal tension and relaxation (Meyer et al., 2011). It is plausible to assume that a viscous muscle would take much longer to adapt to a mechanical stress than a less viscous muscle. Furthermore, muscle $\eta$ may be affected by muscle fatigue (Zhang and Rymer, 2001), which is important, because higher $\eta$ may be related to reductions in muscle fiber relaxation and the detachment rate of cross-bridges, affecting muscle function. Changes in muscle $\eta$ with fatigue may affect the natural capacity of muscles to absorb mechanical shock and prevent overly sudden changes in tension. However, the in vivo characterization of muscle $\eta$ and its modification with fatigue has not been systematically investigated in humans, and current methods are limited.

The ultrasound SWE approach provides an opportunity to quantify the viscoelastic properties of muscle in vivo by assessing both the local shear modulus $\mu$ and viscosity $\eta$ from the frequency-dependent changes of the shear-wave speed (i.e., dispersion) using rheological models (Catheline et al., 2004; Deffieux et al., 2009; Gennisson et al., 2010; Rasool et al., 2018). Shear-wave signal processing is known as shear-wave spectroscopy (SWS). Rheological models are based on the study of both elastic (spring) and viscous (dashpot) elements connected in various series and parallel configurations, assuming specific criteria, including linearity, homogeneity, and isotropy of the muscle tissue. However, this method has been rarely employed to investigate the specific viscoelastic properties of muscle in humans.

Therefore, we aimed to investigate the effects of repeated voluntary maximal isometric contractions of the KE muscles on resting and active shear modulus and viscosity of the VL muscle in vivo immediately after exercise and during recovery. We hypothesized that high-intensity exercise would induce a high level of peripheral fatigue affecting the viscoelastic properties (reduced stiffness and higher viscosity) of the VL as well as the KE force transmission capacity.

\section{MATERIALS AND METHODS}

\section{Participants}

Twelve young males (age: $29.0 \pm 4.5$ years, body mass: $81.6 \pm 7.1 \mathrm{~kg}$, and height: $176.0 \pm 8.0 \mathrm{~cm}$ ) provided their written informed consent and volunteered to participate in the present study. Participants performed regular physical activity, such as strength training, running, and/or cross-training (between 6 and $15 \mathrm{~h} / \mathrm{w}$ ), with no recent history of muscular, joint, or bone disorders or of receiving any medication that could interfere with neuromuscular responses. All volunteers were fully informed of the experimental procedures, aims, and risks and gave their written informed consent before any testing was conducted. Each participant participated in an inclusion session, consisting of a complete medical examination, including the collection of anthropometric data and complete familiarization with the experimental procedures. This study was approved by the scientific leadership of the French Armed Forces Biomedical Research Institute and the local ethics committee (CPP Ile de France VI). All experiments were conducted in accordance with the Helsinki Declaration.

\section{Protocol}

Participants performed an intermittent voluntary fatigue protocol consisting of 6 sets $\times 10$ repetitions of 5-s maximal voluntary isometric contractions (MVICs) of the KE muscles and 5-s passive recovery periods between repetitions with $10 \mathrm{~s}$ of rest between sets (Figure 1A). The number of contractions was chosen to generate a high level of voluntary strength loss 


\section{VOLUNTARY INTERMITTENT FATIGUE PROTOCOL}

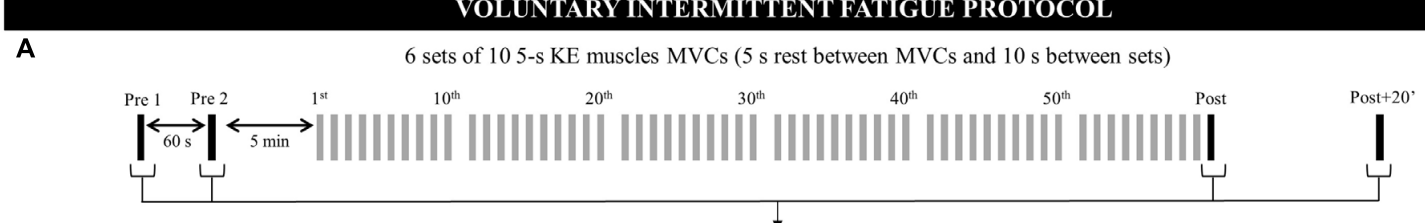

Before fatigue protocol, at the end and during recovery

ELECTRICAL STIMULATION SWE AND SWS MESUREMENTS

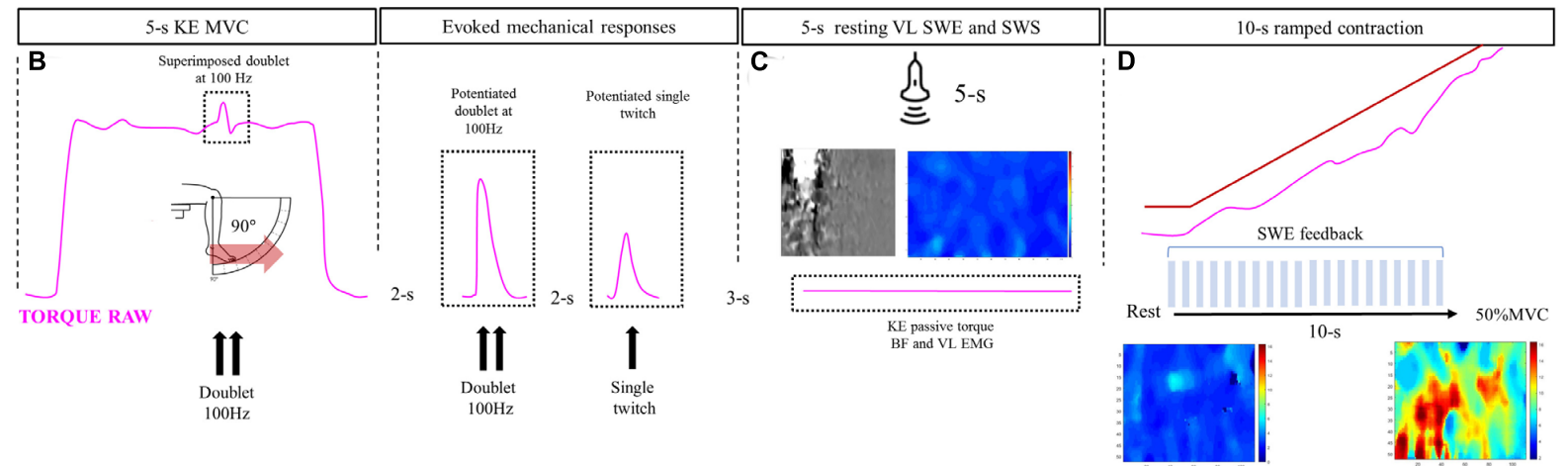

FIGURE 1 | Design of the voluntary intermittent fatigue protocol (top; A), consisting of a series of voluntary force, electrical stimulation, and muscle viscoelasticity measurements performed before and after the fatigue protocol (bottom; B-D). KE, knee extensors; MVC, maximal voluntary contraction; SWE, shear-wave elastography; EMG, surface electromyography; VL, vastus lateralis; BF, biceps femoris; SWS, shear-wave spectroscopy.

and peripheral fatigue, as previously demonstrated (Siracusa et al., 2019). Participants were not informed of the criterion for the end of the task (60 MVCs) but had visual feedback of torque output during the exercise. They were also strongly encouraged by the researcher during the entire fatiguing task and testing. Peripheral fatigue and alterations in the contractile mechanical properties of muscles were determined by delivering double $(\mathrm{Db} 100 \mathrm{~Hz})$ and single (Tw) electrical stimulations to the femoral nerve before, immediately after, and $20 \mathrm{~min}$ after the MVICs at rest (post $+20^{\prime}$; Figures 1A,B). The viscoelastic mechanical properties of the VL muscle (shear $\mu$ and $\eta$ moduli) were evaluated after each electrical-stimulation series (Figure 1C). Then, one 10-s submaximal ramped isometric contraction (from rest to 50\% MVC) of the KE muscle was performed (Figure 1D). VL muscle was chosen because previous studies (Alkner et al., 2000; Place et al., 2007) showed that this muscle is representative of the muscle quadriceps. Moreover, studies showing decreases in muscle stiffness by assessing aponeurosis or muscle tendon junction displacements or by SWE after repeated isometric contractions were made exclusively in the VL muscle (Kubo et al., 2001a; Morel et al., 2019; Siracusa et al., 2019), allowing comparisons with the present study.

\section{Measurements}

\section{Neuromuscular Outcomes}

Participants were comfortably positioned on an adjustable chair with the hip joint flexed at $70^{\circ}\left(90^{\circ}=\right.$ neutral "sitting" position). Before the voluntary fatigue protocol, two 5-s MVICs of the KE were performed, with 60-s passive recovery. The absolute MVIC peak torque was determined as the peak force reached during maximal efforts and was defined as the control "non-fatigued" value. All measurements were taken from the participant's dominant leg (right leg for all participants), which was fixed at $90^{\circ}\left(0^{\circ}=\right.$ knee fully extended $)$. During each MVIC, participants were instructed to grip the seat to stabilize the pelvis. The double pulse (at $100 \mathrm{~Hz}$ ) superimposition technique, based on the interpolated-twitch method (Merton, 1954), enabled us to estimate the maximal voluntary KE activation level. Square-wave pulses with a width of $1 \mathrm{~ms}$ at a maximal voltage of $400 \mathrm{~V}$ were delivered percutaneously to the femoral nerve using an electrical stimulator (Digitimer DS7A, Welwyn Garden City, United Kingdom) at supramaximal intensity. Intensity ranged from 55 to $99 \mathrm{~mA}$, corresponding to $130 \%$ of the optimal intensity (i.e., the intensity at which maximal non-potentiated single twitches started to plateau). Intensities were determined from a progressive recruitment curve. Then, the following parameters were obtained from the potentiated single-twitch response: peak torque $\left(\mathrm{Tw}_{\text {pot }}\right)$, electromechanical delay (EMD), contraction time (CT), half relaxation time (HRT), maximal rate of torque development (MRTD), and maximal rate of torque relaxation (MRTR). Passive KE torque was measured during each SWE measurement at a knee angle of $90^{\circ}$ in order to ensure the relaxed state of the participant and to have an indication of the passive tension which could be interpreted as a global quadriceps muscle-tendon stiffness. The surface electromyography (EMG) signals (root mean square value of $300 \mathrm{~ms}$ ) of the VL and biceps femoris $(\mathrm{BF})$ muscles were recorded to evaluate any muscle 
activity generated during the resting SWE measurements. The bipolar silver chloride surface electrodes (Blue Sensor N-00-S, Ambu, Denmark) were taped lengthwise to the skin over the muscle belly, as recommended by SENIAM (Hermens et al., 2000), with an inter-electrode distance of $20 \mathrm{~mm}$. Electrodes were placed at $2 / 3$ on the line from the anterior spina iliaca superior to the lateral side of the patella for the VL muscle and at $50 \%$ on the line between the ischial tuberosity and the lateral epicondyle of the tibia for the BF muscle. The reference electrode was attached to the patella. Low impedance $(Z<5 \mathrm{k} \Omega)$ at the skinelectrode surface was obtained by shaving, gently abrading the skin with fine-grain sandpaper, and cleaning with alcohol. EMG signals were amplified (Dual Bio Amp ML 135, ADInstruments, Australia) with a bandwidth frequency ranging from 10 to $500 \mathrm{~Hz}$ (common mode rejection ratio $>85 \mathrm{~dB}$, gain $=1,000$ ) and simultaneously digitized together with the torque signals. The sampling frequency was $2 \mathrm{kHz}$. The temperature of the experimental room was controlled $\left(20^{\circ} \mathrm{C}\right)$.

\section{Shear-Wave Elastography Measurements}

An ultrafast ultrasound scanner (Aixplorer version 12.2; Supersonic Imagine, Aix-en-Provence, France) coupled with a linear transducer array (SuperLinear 15-4; Supersonic Imagine, Aix-en-Provence, France) was used in the SWE mode (musculoskeletal preset, penetration, no persistence), as previously proposed (Bercoff et al., 2004). SWE and SWS measurements were carefully standardized. The B-mode ultrasound was first set to determine the optimal transducer location and maximize the alignment between the transducer and the direction of the muscle fascicles. Transducer alignment was considered to be correct when VL muscle fascicles and aponeurosis could be delineated across the image without interruption. The transducer was fixed using a dynamic probe fixation device (with $360^{\circ}$ adjustments, USONO, Eindhoven, Netherlands) placed over the skin, which was coated with a water-soluble transmission gel (Aquasonic, Parker Laboratory, Fairfield, NJ, United States) to ensure acoustic coupling. Then, a fixed-size square region of interest (ROI; $\sim 1.5 \mathrm{~cm}^{2}$ ), i.e., a region in which shear-wave propagation was analyzed within the muscle, was placed in the middle of the B-mode image below the superficial aponeurosis within the VL. A two-dimensional (2D) real-time stiffness ( $\mu$ shear modulus) color map was obtained with a frame rate of $1.5-2 \mathrm{~Hz}$ and a spatial resolution of $1 \mathrm{~mm}^{2} \times 1 \mathrm{~mm}^{2}$, as described above (Figure 1C). Values were averaged over the largest ROI (a 36 × 114 matrix), and the average of the obtained consecutive matrix was used for subsequent analyses for resting and active measurements.

Two types of sequences were used to estimate the shear modulus $\mu$ and shear viscosity $\eta$ of the medium: (i) the conventional SWE sequence to generate a full image of the local shear-wave velocity (SWV) group at rest and during ramped contractions for the shear modulus $\mu$ (Gennisson et al., 2010) and (ii) the SWS sequence to measure the shear-wave dispersion to estimate the muscle shear viscosity $\eta$ (Deffieux et al., 2009). The last sequence was only available for the static condition due to technological limitations. Thus, muscle shear viscosity $\eta$ was assessed only at rest.

\section{Shear-Wave Elastography Sequence}

The SWE technique consists of a transient and remote palpation generated by the radiation force induced by a focused ultrasonic beam (Figure 2A). Each ultrasonic beam generates a remote vibration at different depths that results in the propagation of a transient shear-wave (Bercoff et al., 2004). After generation of the shear-wave, an ultrafast ultrasound imaging sequence is performed to acquire successive raw radio-frequency data at a very high frame rate (up to 20,000 frames/s), contrary to conventional ultrasonography (typically 50 frames/s; Figure 2B). A map of the local shear group velocity was estimated for each of the four pushing lines in each pixel of the resulting image based on the displacement moves using a time-of-flight algorithm. The shear-wave propagation velocity, typically a few meters per second in soft tissue, correlates directly with muscle $\mu$ if the medium is assumed to be purely elastic, homogeneous, and locally isotropic in the imaging plane, which is well-accepted in muscle elastography studies (Bercoff et al., 2004; Catheline et al., 2004). The $\mu$ was obtained as follows:

$$
\mu=\rho . V s^{2}
$$

where $\rho$ is the muscle density $\left(1,000 \mathrm{~kg} / \mathrm{m}^{3}\right)$ and $V_{s}$ is the shearwave speed (in $\mathrm{m} / \mathrm{s}$ ). This equation implicitly neglects viscous effects (assuming the medium to be non-dispersive).

\section{Resting and Active Muscle Stiffness Measurements}

A 5-s SWE sequence was performed at rest for the calculation of the resting VL $\mu$ prior to the active measurements, for which the $2 \mathrm{D}$ real-time color map was maximally homogeneous. Then, the $\mu$ was calculated under the same experimental conditions throughout an incremental isometric task, consisting of a 10s smooth linear force ramp from 0 to $50 \%$ MVIC (adjusted at each time point after exercise: post and post $\left.+20^{\prime}\right)$. A $5 \mathrm{~V}$ TTL signal was triggered at the generation of each SWE image using a PowerLab system (16/30-ML880/P, ADInstruments, Bella Vista, Australia) connected to the ultrasound device to synchronize the SWE measurements with the torque values. During the ramped contractions, the cutoff was set at $50 \%$ MVIC, corresponding to the upper limit, after which the elastography measurement reaches saturation for up to $25 \%$ of the ROI (saturation $=16.329 \mathrm{~m} / \mathrm{s}$ or $800 \mathrm{kPa}$ ), as observed in a previous study (Siracusa et al., 2019). An active muscle stiffness index was then calculated from a linear regression between the mean VL $\mu$ values and $\mathrm{KE}$ absolute torque (slope), which is independent of the level of force. All SWE measurements were transferred to a workstation and analyzed using a MATLAB (MathWorks, Natick, MA, United States) script developed in our laboratory.

\section{Shear-Wave Spectroscopy Sequence}

The SWS sequence, dedicated to the measurement of shearwave dispersion (phase velocity versus frequency), corresponds to the previously proposed sequence (Deffieux et al., 2009). After each pushing beam $(\times 4)$ induced by the ultrasonic probe, the acquired velocity field $u(x, t, z)$ (Figure $2 \mathbf{B}$ ) of the SSI acquisition was averaged along the depth $z$. Then, the $3 \mathrm{D}$ tissue velocity field $u(x, t, z)$ was transformed into a $2 \mathrm{D}$ tissue velocity field 

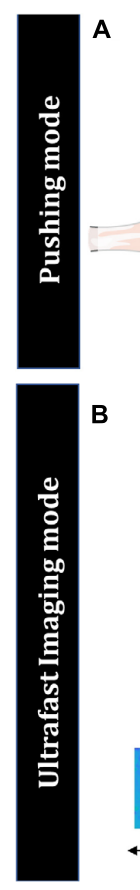

B
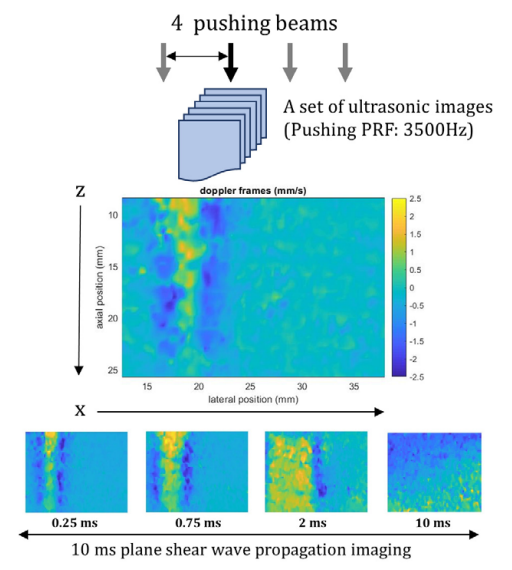

FIGURE 2 | Schema of the imaging setup with the ultrasound transducer (A). Ultrafast imaging mode: the propagation of the planar shear waves of the first pushing beam is shown over $10 \mathrm{~ms}$ (B). Processing of the supersonic imaging data: velocity field of the shear-wave velocity along the fibers (C), relationship between the phase delay (ms) and the distance (mm) (D), and rheological model used to explain the viscoelastic properties of muscle (E).

$u(x, t)$, describing the propagation of the plane wave in the square box along the lateral distance $x$ (Figure 2C). A Fourier transform in the time domain was performed on the velocity field, allowing computation of the phase of the shear wave for each frequency of the bandwidth (between 87.5 and $612.5 \mathrm{~Hz}$ ). Phases were linearly fitted as a function of the lateral distance to obtain the SWV dispersion curve (Figure 2D). These velocity measurements were then related to the viscoelastic properties of muscle using a viscoelastic model (Voigt; Figure 2E). The Voigt model links the applied stress $\sigma$ to the resulting strain $\varepsilon$ with the well-known equation:

$$
\sigma=\mu \varepsilon+\eta \partial \varepsilon
$$

Under the assumption of planar shear-wave propagation, this governing equation leads to the expression of the phase velocity as (Chen et al., 2004):

$$
V \phi(\omega)=\frac{\sqrt{2\left(\mu^{2}+\omega^{2} \eta^{2}\right)}}{\rho\left(\mu+\sqrt{\left(\mu^{2}+\omega^{2} \eta^{2}\right)}\right.}
$$

A non-linear optimization technique, such as the classical Nelder-Mead technique (Nelder and Mead, 1965), makes it possible to estimate both $\mu$ and $\eta$ from the measurement of the dispersion law $\mathrm{V}_{\eta}(\omega)$. We used this approach to calculate a global index of the muscle $\eta$ under passive conditions. One SWS sequence was triggered (Figure 1B), solely at rest, before the SWE measurements to calculate the shear $\eta$. The slopes of the linear regressions obtained between the phase velocity and frequencies were used to calculate the shear-wave dispersion index.

\section{Statistical Analysis}

A sample size of 3 (12 subjects included) was deemed sufficient to achieve high statistical power. This was based on the MVC effect size calculated from our previous study $(n=15$; Siracusa et al., 2019) using the sample size calculator $\mathrm{G}^{*}$ Power 3.1.9.2. The sample size calculation was performed a priori with an $\mathrm{F}$ test for ANOVA: repeated measures, within factors and an effect size of 2.074 , an $\alpha$ error probability of 0.05 , a power of 0.95 , one group and three measurements, a correlation among repeated measures of 0.5 , and a non-sphericity correction e of 1 .

The data were screened for normality of the distribution and homogeneity of variances using the Shapiro-Wilk normality and Levene tests, respectively. Differences in absolute values of the passive $\mu, \eta$, slopes, and neuromuscular outcomes were compared by one-way ANOVA (effect:time). Coefficients of determination $\left(\mathrm{R}^{2}\right)$ were calculated for each linear fitting. Results with a $P$-value $<0.05$ were considered to be significant. For VL $\mu$ and $\eta$, interclass correlation coefficient (ICC; 1,1 case), standard error of the mean (SEM), and coefficient of variation (CV) were also evaluated. Statistical procedures were performed using Statistica 8.0 software (Statsoft, Inc., United States). The results are presented as the means \pm SD for the table, text, and figures.

\section{RESULTS}

\section{Repeatability of Measurements}

For VL $\mu$, ICC, SEM, and CV values varied from 0.93 to 0.95 , 0.4 to $0.7 \mathrm{kPa}$, and from 15.3 to $21 \%$ for the VL muscle. For 
VL $\eta$, ICC, SEM, and CV values varied from 0.94 to $0.95,0.5$ to $0.6 \mathrm{~Pa} \times \mathrm{s}$, and from 20.6 to $46.9 \%$ for the VL muscle.

\section{Neuromuscular Outcomes}

One-way ANOVA showed a significant absolute (relative to the "non-fatigued" control value) time-dependent effect on voluntary and evoked torque $(P<0.001)$. MVC, $\mathrm{Db} 100 \mathrm{~Hz}$, and $\mathrm{Tw}_{\text {pot }}$ decreased by $36.2 \pm 8.7 \%(P<0.01), 34.7 \pm 8.8 \%(P<0.001)$, and $42.5 \pm 11.5 \%(P<0.001)$, respectively, by the end of the exercise phase of the protocol $(P<0.001)$. Mechanical properties of the $\mathrm{Tw}_{\text {pot }}$ (CT, MRTD, HRT, and MRTR) were also altered by fatigue $(P<0.05)$. Only MVC torque and HRT returned to baseline at post +20 '. The voluntary KE activation level was not significantly different at the end of the fatigue protocol or during recovery. Passive KE torque and resting EMG activity of the VL and BF muscles assessed during the SWE measurements remained unchanged during the fatigue protocol and recovery period. All absolute values are presented in Table $\mathbf{1}$.

\section{The Relationship Between Shear-Wave Velocity and Frequency}

We quantified the local shear viscosity $\eta$ from the frequencydependence changes of the SWV (dispersion) using Voigt's model. ANOVA showed a significant time effect for the phase velocity versus frequency relationship $(P<0.001$; Figure 3$)$. With fatigue, the mean of the slope was $682.4 \pm 646.6 \%$ higher immediately after exercise and $360.1 \pm 383.8 \%$ higher at post +20 ' than the pre-exercise values.

\section{Resting Viscosity}

ANOVA showed a significant time-dependent effect for absolute resting VL $\eta(P<0.05$; Figure 5A). Resting VL $\eta$ values increased by the end of the fatigue protocol $(179.0 \pm 123.0 \%, P<0.01)$ but returned to baseline by post +20 '. Individual values for the resting $\eta$ are presented in Figure 5C.

\section{Resting Vastus Lateralis $\mu$ and Active Muscle Stiffness Index}

ANOVA showed a significant time-dependent effect for the resting VL $\mu(P<0.001$; Figure 5A $)$. The resting VL $\mu$ was significantly lower at the end of the exercise $(-57.9 \pm 5.4 \%$, $P<0.001)$ and recovery periods $(-27.0 \pm 10.4 \%, P<0.001)$. With fatigue, the active muscle stiffness index was $23.5 \pm 17.5 \%$ lower immediately after exercise and $1.8 \pm 23.4 \%$ lower at post +20 ' than the pre-exercise values (Figure 4).

\section{DISCUSSION}

The main results of this study were that muscle fatigue induces alterations of the viscoelastic properties of the VL (more compliant and viscous muscle). We observed $\sim 57 \%$ reduction of the resting VL $\mu$ and $\sim 23 \%$ lower active muscle stiffness index (slope) after intermittent maximal isometric $\mathrm{KE}$ contractions. Moreover, we found that muscle $\eta$ increased by $\sim 150 \%$ with fatigue. The viscoelastic properties ( $\eta$ and active muscle stiffness index) progressively returned to baseline values by $20 \mathrm{~min}$ after exercise (at post $+20^{\prime}$ ), contrary to the VL $\mu$ and KEevoked mechanical responses $\left(\mathrm{Db} 100 \mathrm{~Hz}\right.$ and $\left.\mathrm{Tw}_{\text {pot }}\right)$, which remained reduced.

\section{Repeatability of Measurements}

This study is the first to address the interest of $\eta$ on a non-contracted muscle (VL) before and after a series of fatiguing isometric MVCs. Methodological factors such as slight probe motion, compression, variability of the measurement, and the ability of subjects to achieve a fully relaxed state

TABLE 1 | Absolute values for both neuromuscular and viscoelastic outcomes before and after exercise.

\begin{tabular}{|c|c|c|c|c|c|}
\hline & Pre & Post & & Post +20 & \\
\hline KE MVC (Nm) & $336.7 \pm 51.5$ & $213.6 \pm 37.2$ & $\star \star \star ~$ & $310.0 \pm 46.9$ & \\
\hline \multicolumn{6}{|l|}{ Nervous factors } \\
\hline KE VAL (\%) & $88.2 \pm 4.5$ & $80.9 \pm 13.7$ & & $87.5 \pm 4.5$ & \\
\hline \multicolumn{6}{|l|}{ Muscular factors } \\
\hline KE Db100Hz_pot (Nm) & $118.0 \pm 13.8$ & $77.1 \pm 14.4$ & 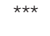 & $97.2 \pm 19.1$ & * \\
\hline KE Tw_pot (Nm) & $80.9 \pm 11.7$ & $46.5 \pm 11.6$ & 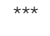 & $51.6 \pm 14.3$ & 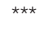 \\
\hline EMD (ms) & $35.4 \pm 10.6$ & $43.6 \pm 13.2$ & & $40.3 \pm 12.2$ & \\
\hline $\mathrm{CT}(\mathrm{ms})$ & $39.6 \pm 8.3$ & $46.4 \pm 10.4$ & * & $42.6 \pm 9.7$ & * \\
\hline MRTD (Nm.s) & $1.8 \pm 0.4$ & $1.0 \pm 0.3$ & 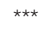 & $1.1 \pm 0.3$ & 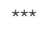 \\
\hline $\mathrm{HRT}(\mathrm{ms})$ & $97.2 \pm 40.7$ & $108.0 \pm 44.1$ & * & $79.8 \pm 26.1$ & \\
\hline MRTR (Nm.s) & $6.5 \pm 1.6$ & $3.4 \pm 1.3$ & 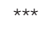 & $2.8 \pm 1.1$ & *** \\
\hline Shear viscosity (Pa.s) & $6.5 \pm 4.3$ & $14.9 \pm 6.6$ & $\star \star$ & $8.9 \pm 4.0$ & \\
\hline Shear elastic modulus (kPa) & $8.8 \pm 1.4$ & $5.2 \pm 0.5$ & 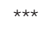 & $8.2 \pm 1.2$ & $\star \star \star ~$ \\
\hline \multicolumn{6}{|c|}{ Resting neuromuscular outcomes } \\
\hline KE Passive Torque (Nm) & $0.3798 \pm 0.1641$ & $0.7937 \pm 0.8977$ & & $0.4519 \pm 0.2442$ & \\
\hline VL resting RMS (mV) & $0.0043 \pm 0.0085$ & $0.0034 \pm 0.0058$ & & $0.0030 \pm 0.0045$ & \\
\hline BF resting RMS (mV) & $0.0015 \pm 0.0002$ & $0.0015 \pm 0.0002$ & & $0.0016 \pm 0.0003$ & \\
\hline
\end{tabular}

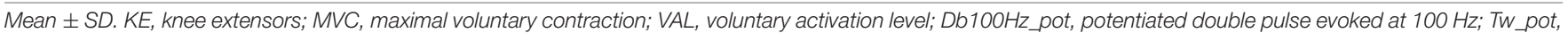

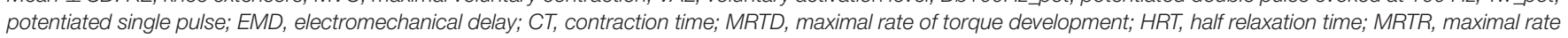
of torque relaxation; $V L$, vastus lateralis; $B F$, biceps femoris; $R M S$, root mean square. ${ }^{*} P<0.05$, ${ }^{* *} P<0.01$, and ${ }^{* * *} P<0.001$, significantly different from pre-exercise. 

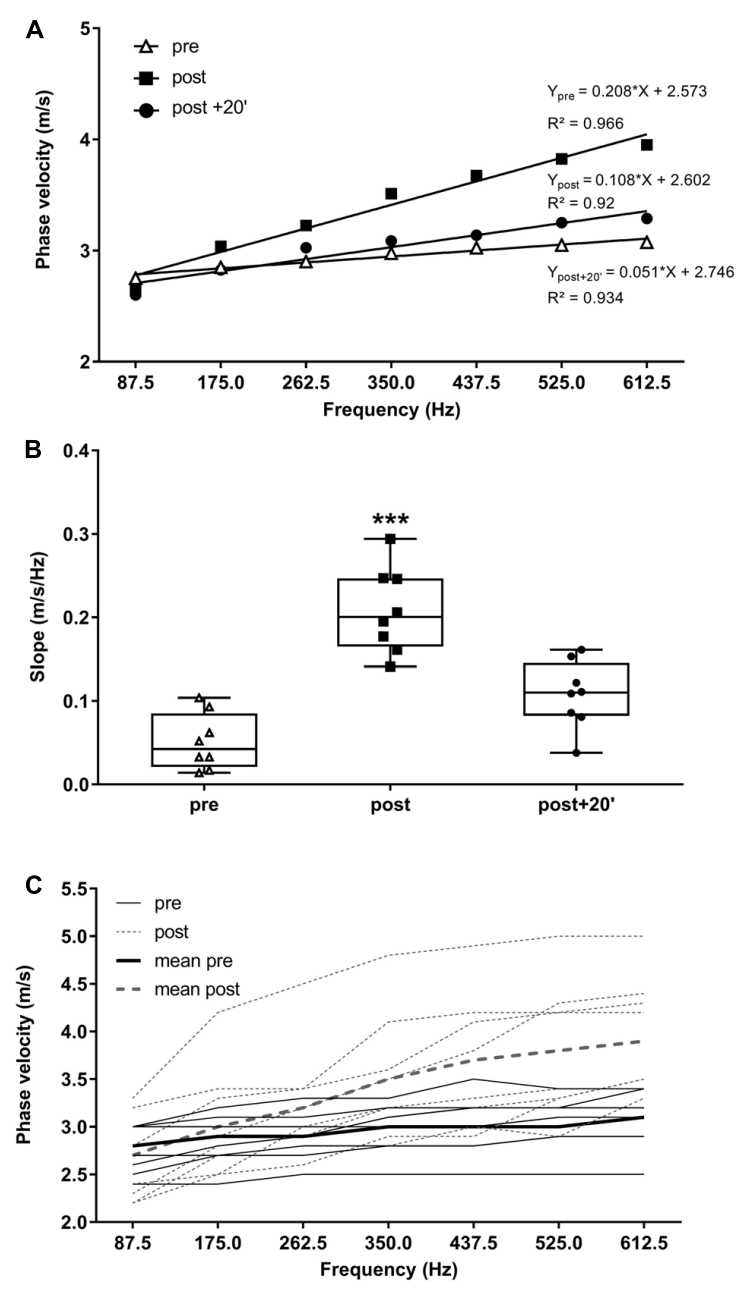

FIGURE 3 | Phase velocity $(\mathrm{m} / \mathrm{s})$ along the fibers as a function of frequency $(\mathrm{Hz})$ at pre, post, and post $+20^{\prime}$. Regressions equations and correlation coefficients are presented for each relationship (A). Mean of the slopes for each time point and individual values. Mean \pm SD (B,C, respectively). ${ }^{* * *} P<0.001$, significant differences from pre-exercise (pre) values.

were meticulously controlled to ensure good reliability of the measurements (Lacourpaille et al., 2012; Eby et al., 2013). There was little variability of the measurements before and after the fatigue protocol. The reproducibility values were obtained using five images (VL $\mu$ ) and two sequences (VL $\eta$ ) at each point. For VL $\mu$, ICC, SEM, and CV values varied from 0.93 to $0.95,0.4$ to $0.7 \mathrm{kPa}$, and from 15.3 to $21 \%$ for the VL muscle. We achieved this level of reproducibility by positioning a dynamic probe fixation system over the skin onto the VL muscle (Siracusa et al., 2019), avoiding any muscle deformation. For VL $\eta$, ICC, SEM, and CV values varied from 0.94 to $0.95,0.5$ to $0.6 \mathrm{~Pa} \times \mathrm{s}$, and from 20.6 to $46.9 \%$ for the VL muscle. This level of reproducibility is slightly higher than $\mu$ possibly due to the anisotropy of muscle tissue. In addition, the VL and BF resting RMS and passive torque did not change during the SWE recordings (Table 1), verifying that the participants were in a relaxed state.
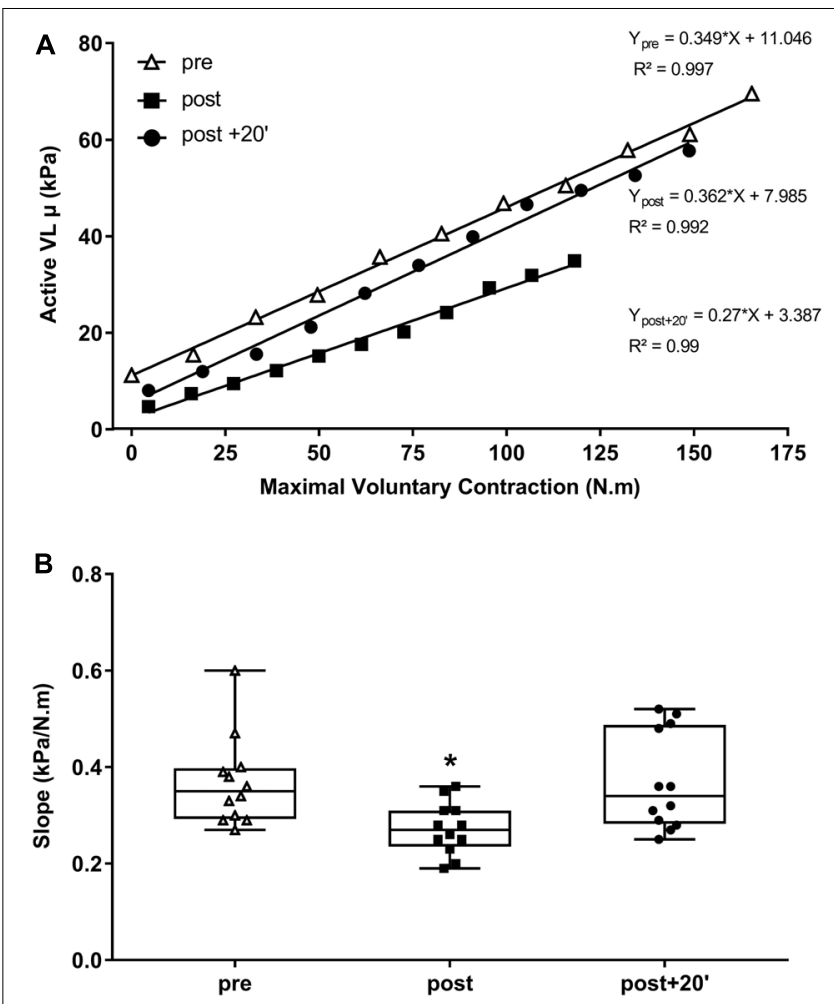

FIGURE 4 | Active vastus lateralis (VL) muscle shear modulus $(\mu)$ and absolute torque relationship of the knee extensor muscles evaluated before and after exercise and during the recovery period (post +20 '). Regression equations and correlation coefficients are presented for each relationship (A). The mean of the slope for each time point and individual values are given (B). Mean $\pm \mathrm{SD}$ (B). ${ }^{*} P<0.05$, significant differences from pre-exercise (pre) values.

\section{Viscoelastic Properties of Muscles After High-Intensity Fatiguing Exercise Muscle Stiffness}

It is well-known that high-intensity exercise induces high peripheral fatigue (Lattier et al., 2004). Peripheral fatigue is generally characterized by altered E-C coupling and calcium kinetics (release and sensitivity), lower intrinsic force and fewer cross-bridges, increased concentrations of inorganic phosphate, etc. (Allen et al., 2008). Moreover, it is classically represented by a reduction in the magnitude of evoked responses (e.g., single twitch) and changes in their mechanical properties (e.g., higher contraction time, lower twitch relaxation time, and velocity) as observed in the present study.

Previous studies have also shown that repeated isometric fatiguing contractions reduce muscle-tendon stiffness, which decreases the force transmission capacity (Kubo et al., 2001a,b; Bojsen-Moller et al., 2003). However, the approach used in these previous studies (i.e., tracking of the muscle-tendon junction) may not be ideal as changes in stiffness mainly reflect modifications of the tendon properties rather than those of muscle. It has been largely demonstrated that SWE provides a reliable quantitative measurement of individual muscle-tissue 

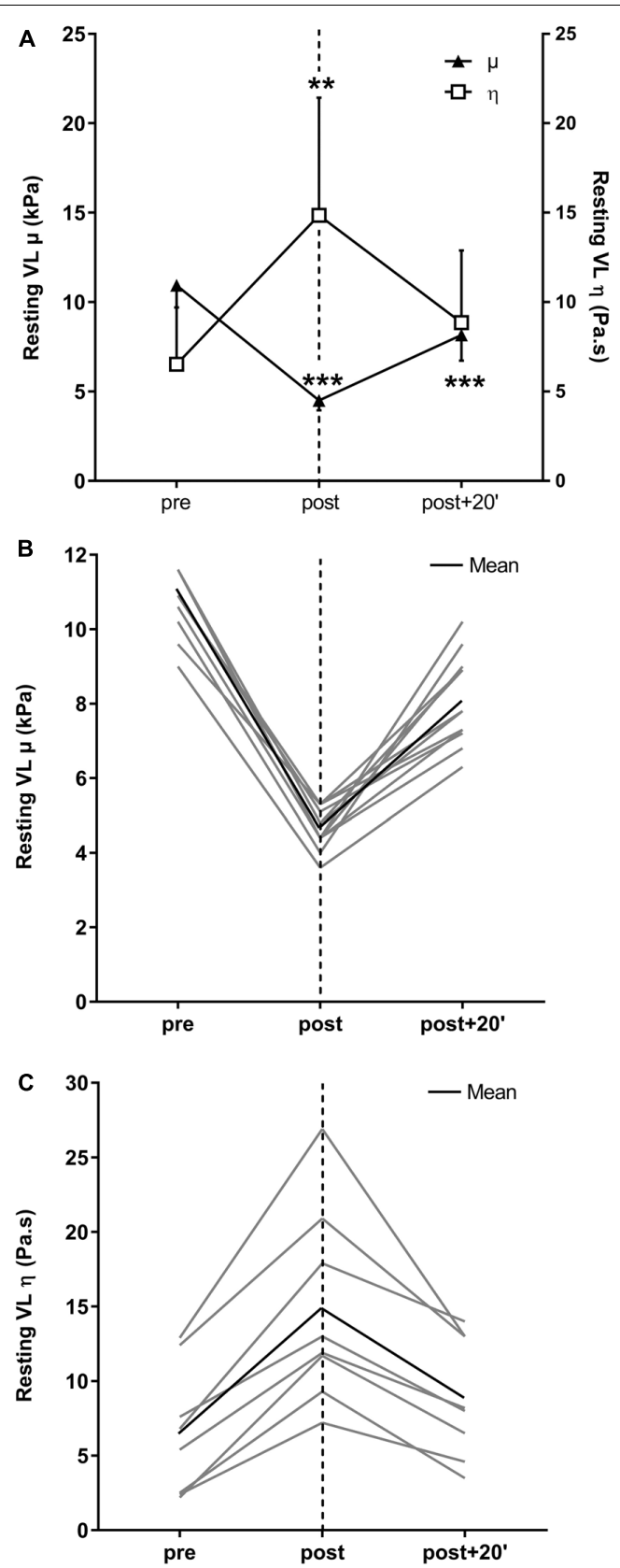

FIGURE 5 | Shear elasticity $(\mu)$ and viscosity $(\eta)$ of the vastus lateralis $(V L)$ muscle extracted from the group velocity and Voigt's model using the dispersion measurement (A). Individual values for $\mu$ (B) and $\eta$ (C). Mean $\pm \mathrm{SD}$. ${ }^{\star \star} P<0.01$ and ${ }^{\star \star \star} P<0.001$, significant differences from pre-exercise (pre) values.

stiffness (Lacourpaille et al., 2012; Brandenburg et al., 2015; Andonian et al., 2016). Moreover, there is a strong linear relationship between individual muscle force (passive and active) and muscle $\mu$ (Bouillard et al., 2011), providing an accurate index of individual muscle force, even when muscle fatigue occurs (Bouillard et al., 2012b).
It has been recently reported that $\mathrm{VL} \mu$ is significantly reduced after maximal $(-34.7 \pm 6.7 \%$ at rest; Siracusa et al., 2019) and submaximal ( $-22 \pm 7 \%$ during contractions; Morel et al., 2019) voluntary fatiguing isometric contractions of the $\mathrm{KE}$ muscles. Andonian et al. (2016) showed a decrease ( $-8 \%)$ in resting $\mu$ of the quadriceps muscle (VL, RF, and VM) after ultraendurance running, which was still reduced more than $48 \mathrm{~h}$ $(\sim-5 \%)$ after exercise. These results are in accordance with those observed in the present study, showing that resting VL $\mu$ was significantly reduced immediately after $(-57.9 \pm 5.4 \%)$ and $20 \mathrm{~min}$ after exercise (post +20 '). However, our results are in contrast to a previous study (Akagi et al., 2019), which showed an increase in resting VL $\mu(21.7 \pm 32.4 \%)$ after a low-intensity prolonged fatiguing task of the KE muscles. Akagi et al. (2017) suggested that resting muscle stiffness could be higher after prolonged fatiguing task due to muscle failure to fully relax and cramps. The well-known task dependency of fatigue needs to be considered to better understand discrepancies concerning changes in muscle stiffness and fatigue. The VL active muscle stiffness index (slope) was also reduced with fatigue $(-27.0 \pm 10.4 \%$ relative to baseline). These results are in accordance with those showing $\sim 12 \%$ reduction of active stiffness of the VL after sustained isometric (Morel et al., 2019), dynamic (Vigreux et al., 1980; Hunter and Kearney, 1983), and intermittent isometric (submaximal) contractions (Zhang and Rymer, 2001). There are several possible explanations for these phenomena. It is possible that muscle fatigue induced a decrease in muscle stiffness of the contractile elements due to the inability of sarcomere cross-bridges to generate as much net force as they produced before fatigue for the same level of external stretch. This was confirmed by Morel et al. (2019), who showed that the VL shear modulus was significantly reduced ( $\sim 16 \%$ lower) at a matched level of absolute force (40\% MVIC; from Pre-) after submaximal sustained isometric contractions $(\sim 15 \mathrm{~min}$ at $60 \%$ MVIC). However, the shear modulus was not assessed at a given absolute torque level after exercise in this study, limiting the conclusions.

Another possible mechanism allowing to explain the reduction in muscle stiffness with fatigue is the modification of the intramuscular temperature with repeated maximal effort, which may also induce a decrease in muscle stiffness. Indeed, a previous study suggested that the increased muscle temperature might contribute to $20-25 \%$ of the EMD elongation found during the fatiguing intermittent exercise (Zhou et al., 1998). As well as the force transmission capacities of muscles may depend on both mechanical and electrochemical processes (membrane excitability, muscle fiber conduction velocity), it has been suggested that any alteration on the electromechanical properties of the skeletal muscle is mainly associated with elastic rather than electrochemical processes (Ce et al., 2013, 2015). Second, the elongation of the connective structures, with fatigue could be attributable to an acute change in the arrangement of the collagen fibers (Stromberg and Wiederhielm, 1969) or alteration of the viscous properties of the intramuscular connective tissue as a result of increased muscle temperature (Strickler et al., 1990). Third, the ratio of force to stiffness cross-bridge may vary in the presence of fatigue induced by repeated contractions. Indeed, it 
has been suggested that stiffness at the sarcomere level is related to the myofilament mechanical properties themselves (Edman, 2009; Colombini et al., 2010). As such, several studies have demonstrated that the attached myosin heads include both the force-generating and the non-force-generating heads (Tsaturyan et al., 2011), with the latter contributing to the stiffness but not to force generation. Finally, the myosin power stroke does not only produce force in the axis of shortening but a radial component also exists (i.e., orthogonal to the long axis of the myofilaments; Williams et al., 2010). This radial component varies with the lattice spacing (i.e., the radial spacing between the contractile filaments), which is influenced by the osmotic pressure as a result of metabolic by-product accumulation during repeated contractions (Rapp et al., 1998). Further studies are needed to bring new insights into the mechanisms that may be involved in the decrease in active and passive muscle stiffness due to fatigue in both radial and longitudinal axes.

The results of the first part of our study confirm that repeated maximal fatiguing exercise induces a reduction in muscle stiffness (a decrease in both the resting and active $\mu$ ), which could be responsible, at least in part, for the deficit in the capacity of force transmission (highlighted by the increased CT and reduced MRTD) with fatigue. Conversely, we found that there were no significant changes in $\mathrm{KE}$ passive torque, despite the reduction in VL muscle stiffness. It could be that muscular changes were too low to affect passive torque, especially at short or neutral muscle length (knee angle of $\leq 90^{\circ}$ ). Indeed, Lacourpaille et al. (2014) showed that changes in shear elastic modulus of biceps brachii was higher at long muscle length than short muscle length after $1 \mathrm{~h}$ of exercise. Moreover, it is possible that muscle fatigue may have induced a change in load sharing among the KE muscles, with a greater extent of fatigue in the VL muscle affecting its intrinsic force. For example, Bouillard et al. (2014) reported that the VL $\mu$ evaluated during a matched sustained submaximal isometric contraction of the KE muscles was lower after a fatiguing exercise, without changes in the other heads of the quadriceps, partially confirming this hypothesis.

\section{Muscle Viscosity ( $\eta$ )}

Studies on the viscoelasticity of muscle have mainly focused on characterizing elastic behavior, largely neglecting the viscous component. However, it is known that severe muscle fatigue may induce an increase in joint $\eta$ (Zhang and Rymer, 2001). It is plausible to assume that a viscous muscle would take much longer to adapt to a mechanical stress than a less viscous muscle. A higher $\eta$ after fatiguing exercise may be related to reduced relaxation of the muscle fibers and a lower cross-bridge detachment rate, affecting muscle function (Westerblad and Allen, 1993). This would affect the natural capacity of muscles to absorb mechanical shocks and prevent overly sudden changes in tension. Although the relationship between the level of muscle viscosity and the degree of protection is still unknown, we could reasonably suggest that any modification of the steady state of the muscle tissue could affect the optimal functioning of the muscle-tendon unit that may promote injuries.

The estimation of muscle shear viscosity $\eta$ is much more complex than that of the shear modulus $\mu$, as it requires the introduction of different rheological models of the medium that consider frequency dependence of the SWV. Thus, it provides more information on tissue behavior than the simple mapping of the group velocity $\left(\mu=\rho \times \mathrm{Vs}^{2}\right)$. Here, based on frequency dependence changes of the shear-wave speed, we observed higher dispersion (greater slope) of shear-wave speed with fatigue (postexercise) than that at pre-exercise or post +20 '. The mean of the slope was $682.3 \pm 646.5 \%$ higher immediately after exercise and $360.1 \pm 383.7 \%$ higher at post $+20^{\prime}$ than baseline. These results are in accordance with those obtained by Deffieux et al. (2008), showing that SWS signal processing clearly depicts the viscous phantom as being highly dispersive, represented by a greater slope over the range of frequencies. Based on this relationship, we quantified the local resting VL $\eta$, which increased after fatigue (from $\sim 6.5$ to $\sim 15 \mathrm{~Pa} \times \mathrm{s}$ ) and returned to baseline 20 min after exercise. These results were also accompanied by a prolonged relaxation time and velocity (HRT and MRTR), calculated from the single evoked torque, suggesting that higher $\eta$ may be related to reduced muscle fiber relaxation and a lower crossbridge detachment rate, affecting muscle function. However, the HRT represents a global index of the muscle force of the entire quadriceps and not only that of the VL.

\section{Limitations and Perspectives}

While this study provides scientific evidence of changes in VL $\mu$ and $\eta$ with fatigue, it had methodological limitations: the entire quadriceps complex was not evaluated, limiting the interpretation of our results concerning other synergistic muscles (rectus femoris and vastus medialis), and the mathematical model used to estimate the elastic and viscous moduli assumes homogeneity and isotropy of the medium, whereas muscle is an anisotropic material. Anisotropy, assumed to be transverse isotropic in muscles, was not significant, as we investigated the imaging plane in the axis of the fibers. Moreover, no intermediate recovery measures were made, limiting interpretation about the time course of the viscoelastic parameters after the fatigue protocol representing restoration of the force transmission capacities. Finally, after the reproducibility tests, we found relatively high values for CV (>20\%) mainly for VL $\mu$ and $\eta$. Generally, the criteria for $\mathrm{CV}$ is considered to be $<15 \%$ compared to the criteria for example for ultrasound studies (Van Hooren et al., 2020). This high level of CV is possibly due to ultrasound probe positioning which could have been moved during contractions despite the probe fixation system used or anisotropy of the muscle tissue. Moreover, in the present study, muscle viscosity was estimated after delivering four pushing beams by the ultrasonic probe at different levels of the region of interest as presented in Figure 2B. It is possible that the high CV values obtained were explained by the difference in the lateral distance (time of propagation) of each pushing beam affecting the analyzed velocity of the shear waves and its attenuation at different frequencies. However, VL $\eta$ seems to be an interesting measure because it is sensitive to mechanical changes with fatigue despite the high level of CV (Figure 5; $P<0.01$ ). For all participants, VL $\eta$ values increased after the end of the fatigue protocol and decreased with the recovery (post +20$)$. A new SWS sequence needs to be developed in order to deliver several pushing beams in the left side of the ultrasound probe in order to ensure reproducibility of values. 
Although these results must be interpreted with caution, they show promise in helping to gain a better understanding of the changes in the viscoelastic properties of muscle with fatigue. Moreover, studies focused on the mechanical properties of mechanical evoked responses coupled to B-mode ultrasound (ultra-fast sequences, very high frame rate) and their relationship to muscle stiffness and viscosity are required to gain insights about modifications in the force transmission capacity of individual muscles and fatigue.

\section{CONCLUSION}

We have shown that VL muscle stiffness is strongly reduced by fatigue, both at rest and when active, reflecting higher muscle compliance. Moreover, such higher muscle compliance was accompanied by a greater dispersion of the shear waves, reflecting a more viscous muscle. In vivo evaluation of tissue viscosity may play a significant role in introducing frequency-dependent changes (dispersion) in muscle tissue, and parallel changes in elasticity may significantly alter the mechanical behavior of the tissue. The quantitative study of the viscoelastic properties of muscle may have important implications in sports medicine, as well as other fields of medicine, for better understanding the development of muscle fatigue and various pathological conditions, such as muscle dystrophy, motor neuron diseases, and inflammatory and metabolic myopathies.

\section{DATA AVAILABILITY STATEMENT}

The datasets generated from this study are available on request to the corresponding author.

\section{REFERENCES}

Akagi, R., Fukui, T., Kubota, M., Nakamura, M., and Ema, R. (2017). Muscle shear moduli changes and frequency of alternate muscle activity of plantar flexor synergists induced by prolonged low-level contraction. Front. Physiol. 8:708. doi: 10.3389/fphys.2017.00708

Akagi, R., Sato, S., Yoshihara, K., Ishimatsu, H., and Ema, R. (2019). Sex difference in fatigability of knee extensor muscles during sustained low-level contractions. Sci. Rep. 9:16718. doi: 10.1038/s41598-019-53 $375-\mathrm{z}$

Alkner, B. A., Tesch, P. A., and Berg, H. E. (2000). Quadriceps EMG/force relationship in knee extension and leg press. Med. Sci. Sports Exerc. 32, 459463.

Allen, D. G., Lamb, G. D., and Westerblad, H. (2008). Skeletal muscle fatigue: cellular mechanisms. Physiol. Rev. 88, 287-332. doi: 10.1152/physrev.00015. 2007

Andonian, P., Viallon, M., Le Goff, C., De Bourguignon, C., Tourel, C., Morel, J., et al. (2016). Shear-Wave elastography assessments of quadriceps stiffness changes prior to, during and after prolonged exercise: a longitudinal study during an extreme mountain ultra-marathon. PLoS One 11:e0161855. doi: 10. 1371/journal.pone.0161855

Bercoff, J., Tanter, M., and Fink, M. (2004). Supersonic shear imaging: a new technique for soft tissue elasticity mapping. IEEE Trans. Ultrason. Ferroelectr. Freq. Control 51, 396-409. doi: 10.1109/tuffc.2004.129 5425

Bojsen-Moller, J., Hansen, P., Aagaard, P., Kjaer, M., and Magnusson, S. P. (2003). Measuring mechanical properties of the vastus lateralis tendon-aponeurosis

\section{ETHICS STATEMENT}

This study was approved by the scientific leadership of the French Armed Forces Biomedical Research Institute and the local Ethics Committee (CPP Ile de France VI). All experiments were conducted in accordance with the Helsinki Declaration Schuklenk, 2001.

\section{AUTHOR CONTRIBUTIONS}

EC, KC, JS, and SG-V designed the study, performed the analysis and interpretation of the data, and drafted the manuscript. AM, $\mathrm{KC}, \mathrm{CB}$, JS, and SG-V participated in the data collection. J-LG, $\mathrm{LP}, \mathrm{MO}, \mathrm{AM}, \mathrm{KC}$, and $\mathrm{CB}$ critically revised the manuscript.

\section{FUNDING}

This work was supported by the Service de Santé des Armées.

\section{ACKNOWLEDGMENTS}

The authors would like to thank Nathalie Koulmann for initiating this project and Stéphane Baugé, Stéphanie Bourdon, Philippe Colin, and Benoit Lepetit for their technical support during the experiments. We thank MD Oliver Nespoulous for his medical support. Finally, we thank Antoine Nordez and Vincent Martin for helping us to improve this manuscript.

complex in vivo by ultrasound imaging. Scand. J. Med. Sci. Sports 13, 259-265. doi: 10.1034/j.1600-0838.2003.00301.x

Bouillard, K., Hug, F., Guevel, A., and Nordez, A. (2012a). Shear elastic modulus can be used to estimate an index of individual muscle force during a submaximal isometric fatiguing contraction. J. Appl. Physiol. 113, 1353-1361. doi: 10.1152/japplphysiol.00858.2012

Bouillard, K., Nordez, A., Hodges, P. W., Cornu, C., and Hug, F. (2012b). Evidence of changes in load sharing during isometric elbow flexion with ramped torque. J. Biomech. 45, 1424-1429. doi: 10.1016/j.jbiomech.2012.02.020

Bouillard, K., Jubeau, M., Nordez, A., and Hug, F. (2014). Effect of vastus lateralis fatigue on load sharing between quadriceps femoris muscles during isometric knee extensions. J. Neurophysiol. 111, 768-776. doi: 10.1152/jn.00595.2013

Bouillard, K., Nordez, A., and Hug, F. (2011). Estimation of individual muscle force using elastography. PLoS One 6:e29261. doi: 10.1371/journal.pone.0029261

Brandenburg, J. E., Eby, S. F., Song, P., Zhao, H., Landry, B. W., Kingsley-Berg, S., et al. (2015). Feasibility and reliability of quantifying passive muscle stiffness in young children by using shear wave ultrasound elastography. J. Ultrasound Med. 34, 663-670. doi: 10.7863/ultra.34.4.663

Catheline, S., Gennisson, J. L., Delon, G., Fink, M., Sinkus, R., Abouelkaram, S., et al. (2004). Measuring of viscoelastic properties of homogeneous soft solid using transient elastography: an inverse problem approach. J. Acoust. Soc. Am. 116, 3734-3741. doi: 10.1121/1.1815075

Ce, E., Rampichini, S., Agnello, L., Limonta, E., Veicsteinas, A., and Esposito, F. (2013). Effects of temperature and fatigue on the electromechanical delay components. Muscle Nerve 47, 566-576. doi: 10.1002/mus.23627

Ce, E., Rampichini, S., Venturelli, M., Limonta, E., Veicsteinas, A., and Esposito, F. (2015). Electromechanical delay components during relaxation after voluntary 
contraction: reliability and effects of fatigue. Muscle Nerve 51, 907-915. doi: 10.1002/mus.24466

Chen, S., Fatemi, M., and Greenleaf, J. F. (2004). Quantifying elasticity and viscosity from measurement of shear wave speed dispersion. J. Acoust. Soc. Am. 115, 2781-2785. doi: 10.1121/1.1739480

Colombini, B., Nocella, M., Bagni, M. A., Griffiths, P. J., and Cecchi, G. (2010). Is the cross-bridge stiffness proportional to tension during muscle fiber activation? Biophys. J. 98, 2582-2590. doi: 10.1016/j.bpj.2010.02.014

Davis, L. C., Baumer, T. G., Bey, M. J., and Holsbeeck, M. V. (2018). Clinical utilization of shear wave elastography in the musculoskeletal system. Ultrasonography 38, 2-12. doi: 10.14366/usg.18039

Deffieux, T., Gennisson, J. L., Tanter, M., and Fink, M. (2008). Assessment of the mechanical properties of the musculoskeletal system using 2-D and 3-D very high frame rate ultrasound. IEEE Trans. Ultrason. Ferroelectr. Freq. Control 55, 2177-2190. doi: 10.1109/TUFFC.917

Deffieux, T., Montaldo, G., Tanter, M., and Fink, M. (2009). Shear wave spectroscopy for in vivo quantification of human soft tissues visco-elasticity. IEEE Trans. Med. Imaging 28, 313-322. doi: 10.1109/TMI.2008.925077

Eby S. F., Song P., Chen S., Chen Q., Greenleaf J. F., and An K. N. (2013). Validation of shear wave elastography in skeletal muscle. J. Biomech. 46, 2381-2387. doi: 10.1016/j.jbiomech.2013.07.033

Edman, K. A. (2009). Non-linear myofilament elasticity in frog intact muscle fibres. J. Exp. Biol. 212, 1115-1119. doi: 10.1242/jeb.020982

Gennisson, J. L., Deffieux, T., Mace, E., Montaldo, G., Fink, M., and Tanter, M. (2010). Viscoelastic and anisotropic mechanical properties of in vivo muscle tissue assessed by supersonic shear imaging. Ultrasound Med. Biol. 36, 789-801. doi: 10.1016/j.ultrasmedbio.2010.02.013

Hermens, H. J., Freriks, B., Disselhorst-Klug, C., and Rau, G. (2000). Development of recommendations for SEMG sensors and sensor placement procedures. J. Electromyogr. Kinesiol. 10, 361-374. doi: 10.1016/s1050-6411(00)00027-4

Hill, A. V. (1922). The maximum work and mechanical efficiency of human muscles, and their most economical speed. J. Physiol. 56, 19-41. doi: 10.1113/ jphysiol.1922.sp001989

Hunter, I. W., and Kearney, R. E. (1983). Invariance of ankle dynamic stiffness during fatiguing muscle contractions. J. Biomech. 16, 985-991. doi: 10.1016/ 0021-9290(83)90099-4

Kubo, K., Kanehisa, H., and Fukunaga, T. (2001a). Effects of different duration isometric contractions on tendon elasticity in human quadriceps muscles. J. Physiol. 536, 649-655. doi: 10.1111/j.1469-7793.2001.0649c.xd

Kubo, K., Kanehisa, H., Ito, M., and Fukunaga, T. (2001b). Effects of isometric training on the elasticity of human tendon structures in vivo. J. Appl. Physiol. 91, 26-32. doi: 10.1152/jappl.2001.91.1.26

Lacourpaille, L., Hug, F., Bouillard, K., Hogrel, J. Y., and Nordez, A. (2012). Supersonic shear imaging provides a reliable measurement of resting muscle shear elastic modulus. Physiol. Meas. 33, N19-N28. doi: 10.1088/0967-3334/33/ 3/N19

Lacourpaille, L., Nordez, A., Hug, F., Couturier, A., Dibie, C., and Guilhem, G. (2014). Time-course effect of exercise-induced muscle damage on localized muscle mechanical properties assessed using elastography. Acta Physiol. 211, 135-146. doi: 10.1111/apha.12272

Lattier, G., Millet, G. Y., Martin, A., and Martin, V. (2004). Fatigue and recovery after high-intensity exercise part I: neuromuscular fatigue. Int. J. Sports Med. 25, 450-456. doi: 10.1055/s-2004-820939

Mauro, A., and Adams, W. R. (1961). The structure of the sarcolemma of the frog skeletal muscle fiber. J. Biophys. Biochem. Cytol. 10, 177-185. doi: 10.1083/jcb. 10.4.177

Merton, P. A. (1954). Voluntary strength and fatigue. J. Physiol. 123, 553-564. doi: 10.1113/jphysiol.1954.sp005070

Meyer, G. A., Mcculloch, A. D., and Lieber, R. L. (2011). A nonlinear model of passive muscle viscosity. J. Biomech. Eng. 133:091007. doi: 10.1115/1.4004993

Morel, B., Hug, F., Nordez, A., Pournot, H., Besson, T., Mathevon, L., et al. (2019). Reduced Active Muscle Stiffness after Intermittent Submaximal Isometric Contractions. Med. Sci. Sports Exerc. 51, 2603-2609. doi: 10.1249/MSS. 0000000000002080

Moss, R. L., and Halpern, W. (1977). Elastic and viscous properties of resting frog skeletal muscle. Biophys. J. 17, 213-228. doi: 10.1016/s0006-3495(77)85651-8
Nelder, J., and Mead, R. (1965). A simplex method for function minimization. Comput. J. 7, 308-313. doi: 10.1093/comjnl/7.4.308

Place, N., Maffiuletti, N. A., Martin, A., and Lepers, R. (2007). Assessment of the reliability of central and peripheral fatigue after sustained maximal voluntary contraction of the quadriceps muscle. Muscle Nerve 35, 486-495. doi: 10.1002/ mus.20714

Rapp, G., Ashley, C. C., Bagni, M. A., Griffiths, P. J., and Cecchi, G. (1998) Volume changes of the myosin lattice resulting from repetitive stimulation of single muscle fibers. Biophys. J. 75, 2984-2995. doi: 10.1016/s0006-3495(98)77 $739-2$

Rasool, G., Wang, A. B., Rymer, W. Z., and Lee, S. S. M. (2018). Shear waves reveal viscoelastic changes in skeletal muscles after hemispheric stroke. IEEE Trans. Neural. Syst. Rehabil. Eng. 26, 2006-2014. doi: 10.1109/TNSRE.2018.2870155

Siracusa, J., Charlot, K., Malgoyre, A., Conort, S., Tardo-Dino, P. E., Bourrilhon, C., et al. (2019). Resting muscle shear modulus measured with ultrasound shearwave elastography as an alternative tool to assess muscle fatigue in humans. Front. Physiol. 10:626. doi: 10.3389/fphys.2019.00626

Stackhouse, S. K., Reisman, D. S., and Binder-Macleod, S. A. (2001). Challenging the role of $\mathrm{pH}$ in skeletal muscle fatigue. Phys. Ther. 81, 1897-1903. doi: $10.1093 / \mathrm{ptj} / 81.12 .1897$

Strickler, T., Malone, T., and Garrett, W. E. (1990). The effects of passive warming on muscle injury. Am. J. Sports Med. 18, 141-145. doi: 10.1177/ 036354659001800206

Stromberg, D. D., and Wiederhielm, C. A. (1969). Viscoelastic description of a collagenous tissue in simple elongation. J. Appl. Physiol. 26, 857-862. doi: 10.1152/jappl.1969.26.6.857

Tsaturyan, A. K., Bershitsky, S. Y., Koubassova, N. A., Fernandez, M., Narayanan, T., and Ferenczi, M. A. (2011). The fraction of myosin motors that participate in isometric contraction of rabbit muscle fibers at near-physiological temperature. Biophys. J. 101, 404-410. doi: 10.1016/j.bpj.2011.06.008

Van Hooren, B., Teratsias, P., and Hodson-Tole, E. F. (2020). Ultrasound imaging to assess skeletal muscle architecture during movements: a systematic review of methods, reliability, and challenges. J. Appl. Physiol. doi: 10.1152/japplphysiol. 00835.2019 [Epub ahead of print].

Vigreux, B., Cnockaert, J. C., and Pertuzon, E. (1980). Effects of fatigue on the series elastic component of human muscle. Eur. J. Appl. Physiol. Occup. Physiol. 45, 11-17. doi: 10.1007/bf00421196

Westerblad, H., and Allen, D. G. (1993). The contribution of [Ca2+]i to the slowing of relaxation in fatigued single fibres from mouse skeletal muscle. J. Physiol. 468, 729-740. doi: 10.1113/jphysiol.1993.sp019797

Williams, C. D., Regnier, M., and Daniel, T. L. (2010). Axial and radial forces of cross-bridges depend on lattice spacing. PLoS Comput. Biol. 6:e1001018. doi: 10.1371/journal.pcbi.1001018

Zhang, L. Q., and Rymer, W. Z. (2001). Reflex and intrinsic changes induced by fatigue of human elbow extensor muscles. J. Neurophysiol. 86, 1086-1094. doi: 10.1152/jn.2001.86.3.1086

Zhou, S., Carey, M. F., Snow, R. J., Lawson, D. L., and Morrison, W. E. (1998). Effects of muscle fatigue and temperature on electromechanical delay. Electromyogr. Clin. Neurophysiol. 38, $67-73$.

Conflict of Interest: J-LG is a scientific consultant for Supersonic Imagine.

The remaining authors declare that the research was conducted in the absence of any commercial or financial relationships that could be construed as a potential conflict of interest. The authors declare that the results are presented clearly, honestly, and without fabrication, falsification, or inappropriate data manipulation.

Copyright (c) 2020 Chalchat, Gennisson, Peñailillo, Oger, Malgoyre, Charlot, Bourrilhon, Siracusa and Garcia-Vicencio. This is an open-access article distributed under the terms of the Creative Commons Attribution License (CC BY). The use, distribution or reproduction in other forums is permitted, provided the original author(s) and the copyright owner(s) are credited and that the original publication in this journal is cited, in accordance with accepted academic practice. No use, distribution or reproduction is permitted which does not comply with these terms. 